\title{
On clique-inverse graphs of graphs with bounded clique number
}

\author{
Liliana Alcón $^{1,2}$ | Sylvain Gravier ${ }^{3}$ | Claudia L. Sales ${ }^{4}$ | \\ Fabio Protti ${ }^{5}$ (i) | Gabriela Ravenna ${ }^{1,2}$
}

${ }^{1}$ Universidad Nacional de La Plata,

La Plata, Argentina

${ }^{2}$ CONICET, Buenos Aires, Argentina

${ }^{3}$ Université Joseph Fourier, Grenoble,

France

${ }^{4}$ Universidade Federal do Ceará,

Fortaleza, Brazil

${ }^{5}$ Universidade Federal Fluminense,

Niterói, Brazil

\section{Correspondence}

Fabio Protti, Universidade Federal

Fluminense, Niterói, Brazil.

Email: fabio@ic.uff.br

\section{Funding information}

Conselho Nacional de Desenvolvimento Científico e Tecnológico; Fundação

Carlos Chagas Filho de Amparo à

Pesquisa do Estado do Rio de Janeiro;

Consejo Nacional de Investigaciones

Científicas y Técnicas; Coordenação de Aperfeiçoamento de Pessoal de Nível Superior

\begin{abstract}
The clique graph $K(G)$ of $G$ is the intersection graph of the family of maximal cliques of $G$. For a family $\mathcal{F}$ of graphs, the family of clique-inverse graphs of $\mathcal{F}$, denoted by $K^{-1}(\mathcal{F})$, is defined as $K^{-1}(\mathcal{F})=\{H \mid K(H) \in \mathcal{F}\}$. Let $\mathcal{F}_{p}$ be the family of $K_{p}$-free graphs, that is, graphs with clique number at most $p-1$, for an integer constant $p \geq 2$. Deciding whether a graph $H$ is a clique-inverse graph of $\mathcal{F}_{p}$ can be done in polynomial time; in addition, for $p \in\{2,3,4\}, K^{-1}\left(\mathcal{F}_{p}\right)$ can be characterized by a finite family of forbidden induced subgraphs. In Protti and Szwarcfiter, the authors propose to extend such characterizations to higher values of $p$. Then a natural question arises: Is there a characterization of $K^{-1}\left(\mathcal{F}_{p}\right)$ by means of a finite family of forbidden induced subgraphs, for any $p \geq 2$ ? In this note we give a positive answer to this question. We present upper bounds for the order, the clique number, and the stability number of every forbidden induced subgraph for $K^{-1}\left(\mathcal{F}_{p}\right)$ in terms of $p$.

\section{K E Y W O R D S}

clique graph, clique-inverse graph
\end{abstract}

\section{1 | INTRODUCTION}

The clique graph $K(G)$ of $G$ is the intersection graph of the family of maximal cliques of $G$, that is, vertices of $K(G)$ correspond to maximal cliques of $G$, and an edge exists between two vertices in $K(G)$ if and only if the corresponding maximal cliques of $G$ intersect [4]. In the literature, $K$ is often viewed as a unary operator that maps a graph $G$ into its clique graph $K(G)$ [10]. Clique 
graphs have been studied in several aspects, such as: structural characterizations [4,15], complexity of algorithmic recognition [3], images of graph families under the iterated clique operator $[2,5,16]$, convergence/divergence of the clique operator $[5,6,9]$, and theoretical aspects of clique-inverse graphs $[8,12,13,14]$, to name just a few. Several results on clique graphs can be found in the survey [17].

A graph $G$ is a clique-inverse graph of a graph $H$ if $K(G)=H$. Not every graph $H$ admits a clique-inverse graph; this occurs precisely when $H$ is not a clique graph. However, if $H$ admits a clique-inverse graph $G$, then $H$ admits other clique-inverse graphs (for instance, any graph obtained by the addition of a simplicial vertex to any maximal clique of $G$ is also a clique-inverse graph of $H$ ). Thus, the family $K^{-1}(H)=\{G \mid K(G)=H\}$ of the clique-inverse graphs of $H$ either is empty or contains infinitely many graphs.

For a family $\mathcal{F}$ of graphs, the family of clique-inverse graphs of $\mathcal{F}$, denoted by $K^{-1}(\mathcal{F})$, is defined as $K^{-1}(\mathcal{F})=\{G \mid K(G) \in \mathcal{F}\}$. For an integer $p \geq 2$, let $\mathcal{F}_{p}$ be the family of $K_{p}$-free graphs, that is, graphs with clique number at most $p-1$. The problem of deciding whether a graph $G$ is a clique-inverse graph of $\mathcal{F}_{p}$, when $p$ is a constant, can be solved in polynomial time [13]. This can be easily seen by observing that if $G \in K^{-1}\left(\mathcal{F}_{p}\right)$ then each vertex of $G$ is in at most $p-1$ maximal cliques, that is, $G$ contains at most $(p-1) n$ maximal cliques; then, $K(G)$ can be determined in polynomial time by using any polynomial-delay algorithm for the generation of the maximal cliques of a graph, for example [11]. In addition, checking whether the clique number of $K(G)$ is at most $p-1$ amounts to analyzing all the $\left(\begin{array}{c}n^{\prime} \\ p\end{array}\right)$ subsets of $K(G)$ with $p$ vertices, where $n=|V(K(G))|$.

For $p \in\{2,3,4\}$, the family $K^{-1}\left(\mathcal{F}_{p}\right)$ can be characterized by a finite family of forbidden induced subgraphs. Note that a graph in $K^{-1}\left(\mathcal{F}_{2}\right)$ is complete or a disjoint union of cliques, and thus $G \in K^{-1}\left(\mathcal{F}_{2}\right)$ if and only if $G$ contains no $P_{3}$ (a chordless path on three vertices) as an induced subgraph. The cases $p=3$ and $p=4$ are dealt with below.

Theorem 1 (Protti and Szwarcfiter [12]). A graph $G$ is in $K^{-1}\left(\mathcal{F}_{3}\right)$ if and only if $G$ does not contain as an induced subgraph any of the following graphs depicted in Figure 1: $K_{1,3}$, 4-wheel, 4-fan.

Theorem 2 (Protti and Szwarcfiter [12]). A graph $G$ is in $K^{-1}\left(\mathcal{F}_{4}\right)$ if and only if $G$ does not contain as an induced subgraph any of the following graphs depicted in Figure 1: $K_{1,4}$, 4-wheel, 5-wheel, 5-fan, 4-broom, $H_{0}, H_{0}^{*}, Q_{2}$.

Let $G \in K^{-1}\left(\mathcal{F}_{p}\right)$, for some $p \geq 2$, and let $H$ be an induced subgraph of $G$. Clearly, every maximal clique of $H$ is contained in some maximal clique of $G$. Suppose that there are $p$ distinct, pairwise intersecting maximal cliques $C_{1}, \ldots, C_{p}$ in $H$, and let $C_{i}^{\prime}$ be a maximal clique of $G$ such that $C_{i} \subseteq C_{i}^{\prime}, 1 \leq i \leq p$. If $C_{i}^{\prime}=C_{j}^{\prime}$ for distinct indices $i$ and $j$, then, since $H$ is an induced subgraph of $G$, we have that every vertex of $C_{i}$ is adjacent to every vertex of $C_{j}$ in $H$, in contradiction with the fact that $C_{i}$ and $C_{j}$ are maximal cliques of $H$. Thus, $C_{1}^{\prime}, \ldots, C_{p}^{\prime}$ are distinct and pairwise intersecting maximal cliques in $G$, that is, $\omega(K(G)) \geq p$, contradicting the assumption $G \in K^{-1}\left(\mathcal{F}_{p}\right)$. Therefore, no family of $p$ distinct and pairwise intersecting maximal cliques can exist in $H$, and thus $\omega(K(H)) \leq p-1$, that is, $H \in K^{-1}\left(\mathcal{F}_{p}\right)$. This shows that being a member of $K^{-1}\left(\mathcal{F}_{p}\right)$ is an induced-hereditary property, and therefore (see [7]), $K^{-1}\left(\mathcal{F}_{p}\right)$ can be characterized by a family of vertex-minimal graphs $G$ such that $\omega(K(G)) \geq p$. Such vertex-minimal graphs are also called forbidden induced subgraphs or minimal obstructions. 
In [12] the authors propose to extend the characterizations in Theorems 1 and 2 to higher values of $p$. A natural question that then arises is: Is there a characterization of $K^{-1}\left(\mathcal{F}_{p}\right)$ by means of a finite family of minimal obstructions, for any $p \geq 2$ ? More formally, for any $p \geq 2$, let $\mathcal{F}$ orb $\left(K^{-1}\left(\mathcal{F}_{p}\right)\right)$ denote the family of minimal obstructions for a graph $G$ to have $\omega(K(G)) \leq p-1$.

Question Is $\mathcal{F}$ orb $\left(K^{-1}\left(\mathcal{F}_{p}\right)\right)$ a finite family of graphs?

In this note we give a positive answer to this question by means of counting arguments showing that the order of any graph $G \in \mathcal{F}$ orb $\left(K^{-1}\left(\mathcal{F}_{p}\right)\right)$ is bounded above by a quadratic function of $p$. In addition, we give an upper bound for the clique number and the stability number of $G$. The remainder of this paper is organized as follows. In Section 2 we present the main results, and in Section 3 our conclusions.

\section{THE MAIN RESULT}

We say that a graph $G$ is a clique-critical if $K(G) \neq K(G-v)$ for all $v \in V(G)$.

Remark 3. If $G \in \mathcal{F}$ orb $\left(K^{-1}\left(\mathcal{F}_{p}\right)\right)$ then $G$ is clique-critical.

By [1], $G$ is clique-critical if and only if for every vertex $v \in V(G)$, there exists a pair of maximal cliques $C$ and $C^{\prime}$ of $G$ such that either $\{v\}=C \backslash C^{\prime}$ or $\{v\}=C \cap C^{\prime}$. For short, we say that $v$ is the exact intersection or the exact difference between two maximal cliques. In what follows, we will show that for a graph $G \in \mathcal{F}$ orb $\left(K^{-1}\left(\mathcal{F}_{p}\right)\right)$, the maximal cliques that realize the vertices as an exact intersection or as an exact difference can be chosen between the maximal cliques of any given pairwise intersecting family with $p$ members.

Given $C_{1}, C_{2}, \ldots, C_{t}$ distinct maximal cliques of a graph $G, t \geq 2$, we define the following subsets of $C_{1}$ :

$$
\begin{aligned}
I & =\left\{x \in C_{1}: \exists i, j \in\{2, \ldots, t\} \text { s.t. } \quad C_{i} \cap C_{j}=\{x\}\right\} \\
D & =\left\{x \in C_{1} \backslash I: \exists i, j \in\{2, \ldots, t\} \text { s.t. } \quad C_{i} \backslash C_{j}=\{x\}\right\} \\
I^{\prime} & =\left\{x \in\left(C_{1} \backslash I\right) \backslash D: \exists j \in\{2, \ldots, t\} \text { s.t. } \quad C_{1} \cap C_{j}=\{x\}\right\} ; \\
D^{\prime} & =\left\{x \in\left(\left(C_{1} \backslash I\right) \backslash D\right) \backslash I^{\prime}: \exists j \in\{2, \ldots, t\} \text { s.t. } \quad C_{1} \backslash C_{j}=\{x\}\right\} .
\end{aligned}
$$

Lemma 4. If $\mathcal{F}=\left\{C_{1}, C_{2}, \ldots, C_{p}\right\}$ is a family of pairwise intersecting maximal cliques of a graph $G \in \mathcal{F}$ orb $\left(K^{-1}\left(\mathcal{F}_{p}\right)\right)$, then $C_{1}=I \cup D \cup I^{\prime} \cup D^{\prime}$.

Proof. Suppose to obtain a contradiction that there exists a vertex $x$ such that $x \in C_{1} \backslash\left(I \cup D \cup I^{\prime} \cup D^{\prime}\right)$.

For every $i \in\{1,2, \ldots, p\}$, either $C_{i} \backslash\{x\}$ is a maximal clique of $G-x$ or $C_{i} \backslash\{x\}$ is contained in some other maximal clique of $G$. In the former case, we let $C_{i}^{\prime}$ be $C_{i} \mid\{x\}$ (notice in this case that $C_{i}^{\prime}$ is a maximal clique of $G-x$, and it is also a maximal clique of $G$ if and only if $x \notin C_{i}$ ); and, in the latter, we let $C_{i}^{\prime}$ be a maximal clique of $G-x$ containing $C_{i} \backslash\{x\}$ (in this case, $C_{i}^{\prime}$ is both a maximal clique of $G-x$ and a maximal clique of $G$, but it does not belong to $\left\{C_{1}, C_{2}, \ldots, C_{p}\right\}$ because $\left.C_{i} \backslash C_{i}^{\prime}=\{x\}\right)$. 
We claim that if $i \neq j$ then $C_{i}^{\prime} \neq C_{j}^{\prime}$. Indeed, assume they are equal and say $C=C_{i}^{\prime}=C_{j}^{\prime}$. If $C$ is not a maximal clique of $G$ then $x \in C_{i} \cap C_{j}, C_{i}^{\prime}=C_{i} \backslash\{x\}$, and $C_{j}^{\prime}=C_{j} \backslash\{x\}$; hence, $C_{i}=C_{j}$, a contradiction. If $C$ is a maximal clique of $G$, we analyze three cases:

(a) if $x \notin C_{i}$ and $x \notin C_{j}$ then $C_{i}^{\prime}=C_{i}$ and $C_{j}^{\prime}=C_{j}$, implying that $C_{i}=C_{j}$, a contradiction;

(b) if $x$ belongs to only one of $C_{i}$ and $C_{j}$, say $C_{i}$, then $C_{i} \backslash\{x\} \subseteq C_{i}^{\prime}=C=C_{j}^{\prime}=C_{j}$, contradicting the fact that $x \notin I \cup D \cup I^{\prime} \cup D^{\prime}$;

(c) if $x \in C_{i}$ and $x \in C_{j}$ then $C_{i} \backslash\{x\} \subseteq C$ and $C_{j} \backslash\{x\} \subseteq C$, and this implies that $C_{i} \cup C_{j}$ is a clique of $G$, another contradiction.

It follows that $C_{1}^{\prime}, C_{2}^{\prime}, \ldots, C_{p}^{\prime}$ are $p$ maximal cliques of $G-x$. Thus, by hypothesis, they are not pairwise intersecting. Let $C_{i}^{\prime}$ and $C_{j}^{\prime}$ have empty intersection. Since $C_{i} \cap C_{j} \neq \varnothing$, we have that $\left.C_{i} \cap C_{j}=x\right\}$, contradicting the fact that $x \notin I \cup I^{\prime}$.

Theorem 5. If $\mathcal{F}=\left\{C_{1}, C_{2}, \ldots, C_{p}\right\}$ is any family of $p$ pairwise intersecting maximal cliques of $G \in \mathcal{F}$ orb $\left(K^{-1}\left(\mathcal{F}_{p}\right)\right)$ then every vertex of $G$ is the exact intersection or the exact difference between two maximal cliques in $\mathcal{F}$.

Proof. Since $G$ is minimal (ie, no induced subgraph of $G$ has $p$ pairwise intersecting maximal cliques), we have that every vertex of $G$ must belong to some of the cliques in $\mathcal{F}$, that is, $V(G) \cup_{1 \leq i \leq p} C_{i}$. In contrast, given any vertex $x$ of $G$, renumbering the maximal cliques of $\mathcal{F}$ if necessary, we can assume, without loss of generality, that $x \in C_{1}$; thus, by Lemma 4, there exist $C_{i}$ and $C_{j}$ in $\mathcal{F}$ such that $\{x\}=C_{i} \cap C_{j}$ or $\{x\}=C_{i} \backslash C_{j}$.

Corollary 6. If $G \in \mathcal{F}$ orb $\left(K^{-1}\left(\mathcal{F}_{p}\right)\right)$ then $|V(G)| \leq 3\left(\begin{array}{c}p \\ 2\end{array}\right)$ and every stable set of $G$ contains at most $p$ vertices.

Proof. By Theorem 5, every vertex is the exact intersection or the exact difference between two of $p$ given maximal cliques; thus, $|V(G)| \leq 3\left(\begin{array}{c}p \\ 2\end{array}\right)$. In addition, since no two vertices of a stable set belong to a same clique, we have that the stability number of $G$ is at most $p$.

Corollary 7. For every $p \geq 2, \mathcal{F}$ orb $\left(K^{-1}\left(\mathcal{F}_{p}\right)\right)$ is finite.

Next lemma holds for any graph. It will be used together with Lemma 9 to bound the size of the cliques of any $G \in \mathcal{F}$ orb $\left(K^{-1}\left(\mathcal{F}_{p}\right)\right)$.

Lemma 8. Let $C_{1}, C_{2}, \ldots, C_{t}$ distinct maximal cliques of a graph $G$. If $t \geq 4$ and $C_{1}=I \cup D \cup I^{\prime} \cup D^{\prime}$ then

$$
\left|C_{1}\right| \leq\left(\begin{array}{c}
t-1 \\
2
\end{array}\right)+1
$$

Proof. Let $C_{1}=I \cup D \cup I^{\prime} \cup D^{\prime}$ and assume $\left|C_{1}\right| \geq 4$, otherwise the proof is trivial. For every vertex $x \in I$ (resp., $x \in D$ ) choose a pair of elements $i, j \in\{2, \ldots, t\}$ such that $C_{i} \cap C_{j}=\{x\}$ (resp., $C_{i} \backslash C_{j}=\{x\}$ ), and let $I_{x}=\{i, j\}$ (resp., $D_{x}=\{i, j\}$ ). 
For every vertex $x \in I^{\prime}$ (resp., $x \in D^{\prime}$ ), choose an element $j \in\{2, \ldots, t\}$ such that $C_{1} \cap C_{j}=\{x\}$ (resp., $C_{1} \backslash C_{j}=\{x\}$ ), and let $I_{x}^{\prime}=\{j\}$ (resp., $D_{x}^{\prime}=\{j\}$ ).

Then the following statements easily hold.

(1) If $x$ and $y$ belong to $I$ then $I_{x} \neq I_{y}$.

(2) If $x$ and $y$ belong to $D$ then $D_{x} \neq D_{y}$. Indeed, assume $D_{x}=D_{y}=\{i, j\}$. Then, without loss of generality, $\{x\}=C_{i} \backslash C_{j}$ and $\{y\}=C_{j} \backslash C_{i}$. Therefore, $C_{i} \backslash\{x\} \subseteq C_{j}$, and thus $y$ is adjacent to all the vertices of $C_{i} \backslash\{x\}$. Since, in addition, $y$ is adjacent to $x$ because both vertices belong to $C_{1}$, we have that $C_{i} \cup\{y\}$ is a clique of $G$, contradicting the fact that $C_{i}$ is a maximal clique.

(3) If $x \in I$ and $y \in D$ then $I_{x} \neq D_{y}$. Indeed, assume $I_{x}=D_{y}=\{i, j\}$, with $i$ and $j$ different from 1. Then $\{x\}=C_{i} \cap C_{j}$ and, without loss of generality, $\{y\}=C_{i} \backslash C_{j}$; thus, $C_{i}=\{x, y\}$, which implies the contradiction $C_{i} \subseteq C_{1}$.

(4) If $x$ and $y$ belong to $I^{\prime}$ then $I_{x}^{\prime} \neq I_{y}^{\prime}$. Let $I_{x}^{\prime}=\{i\}$ and $I_{y}^{\prime}=\{j\}$. Then there is no vertex $z \in I$ such that $I_{z}=\{i, j\}$, and there is no vertex $w \in D$ such that $D_{w}=\{i, j\}$, because $C_{i} \cap C_{j} \cap C_{1}=\varnothing, x \in C_{i} \backslash C_{j}$, and $y \in C_{j} \backslash C_{i}$.

(5) If $x$ and $y$ belong to $D^{\prime}$ then $D_{x}^{\prime} \neq D_{y}^{\prime}$. Let $D_{x}^{\prime}=\{i\}$ and $D_{y}^{\prime}=\{j\}$. Then there is no vertex $z \in I$ such that $I_{z}=\{i, j\}$, and there is no vertex $w \in D$ such that $D_{w}=\{i, j\}$, because $\left|C_{i} \cap C_{j}\right|>1$ (otherwise, $\left|C_{1}\right|=3$ ), $x \in C_{j} \backslash C_{i}$, and $y \in C_{i} \backslash C_{j}$.

(6) If $x \in I^{\prime}$ and $y \in D^{\prime}$ then $I_{x}^{\prime} \neq D_{y}^{\prime}$. Indeed, if $I_{x}^{\prime}=D_{y}^{\prime}=\{i\}$ then $\{x\}=C_{1} \cap C_{i}$ and $\{y\}=C_{1} \backslash C_{i}$, that is, $C_{1}=\{x, y\}$, implying the contradiction $\left|C_{1}\right|=2<4$. Let $I_{x}^{\prime}=\{i\}$ and $D_{y}^{\prime}=\{j\}$. Then there is no vertex $z \in I$ such that $I_{z}=\{i, j\}$, and there is no vertex $w \in D$ such that $D_{w}=\{i, j\}$, because $x \in C_{i} \cap C_{j}, C_{1} \cap\left(C_{i} \backslash C_{j}\right)=\varnothing$, and $\left|C_{j} \backslash C_{i}\right| \geq 2$.

Therefore, if the cardinality of the sets $I, D, I^{\prime}$ and $D^{\prime}$ are denoted by $n_{I}, n_{D}, n_{I^{\prime}}$, and $n_{D^{\prime}}$, respectively, we have the following.

By (1), (2), and (3),

$$
n_{I}+n_{D} \leq\left(\begin{array}{c}
t-1 \\
2
\end{array}\right)
$$

And adding (4), (5), and (6):

$$
n_{I}+n_{D} \leq\left(\begin{array}{c}
t-1 \\
2
\end{array}\right)-\left(\begin{array}{c}
n_{D^{\prime}} \\
2
\end{array}\right)-\left(\begin{array}{c}
n_{I^{\prime}} \\
2
\end{array}\right)-n_{I^{\prime}} n_{D^{\prime}}
$$

Let $\left(\begin{array}{l}a \\ b\end{array}\right)=0$ whenever $a<b$.

Since $C_{1}=I \cup D \cup I^{\prime} \cup D^{\prime}$, by inequality (2), we have:

$$
\begin{aligned}
\left|C_{1}\right|= & n_{I}+n_{D}+n_{I^{\prime}}+n_{D^{\prime}} \leq\left(\begin{array}{c}
t-1 \\
2
\end{array}\right)-\left(\begin{array}{c}
n_{D^{\prime}} \\
2
\end{array}\right)-\left(\begin{array}{c}
n_{I^{\prime}} \\
2
\end{array}\right) \\
& -n_{I^{\prime}} n_{D^{\prime}}+n_{I^{\prime}}+n_{D^{\prime}}=\left(\begin{array}{c}
t-1 \\
2
\end{array}\right) \\
& +\frac{1}{2}\left(3\left(n_{I^{\prime}}+n_{D^{\prime}}\right)-\left(n_{I^{\prime}}+n_{D^{\prime}}\right)^{2}\right) .
\end{aligned}
$$



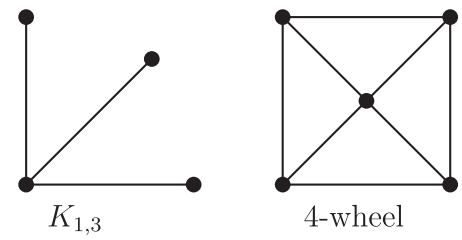

4-wheel
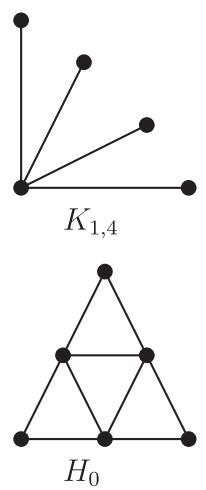

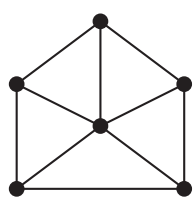

5-wheel

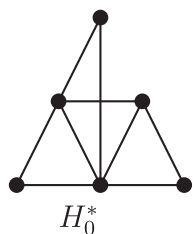

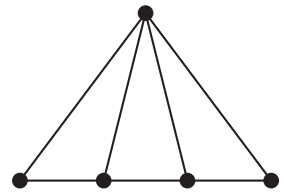

4-fan

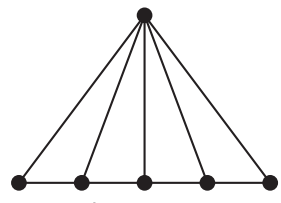

5-fan

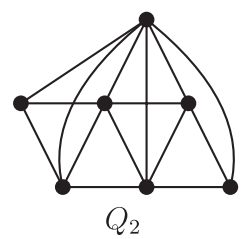

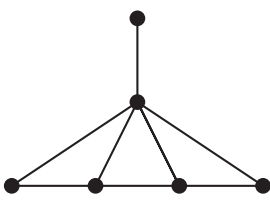

4-broom

FIG URE 1 Graphs in the statements of Theorems 1 and 2

Since $n_{I^{\prime}}+n_{D^{\prime}}$ is a nonnegative integer, it is easy to check that $3\left(n_{I^{\prime}}+n_{D^{\prime}}\right)-\left(n_{I^{\prime}}+n_{D^{\prime}}\right)^{2} \leq 2$. Thus the proof is complete.

We remark that Lemma 8 fails for the case $t=3$. Consider the graph 4 -fan in Figure 1 , and let $C_{1}$ be a triangle of it containing a simplicial vertex. Then $C_{1}=I \cup D \cup I^{\prime} \cup D^{\prime}$, but $\left|C_{1}\right|=3>\left(\begin{array}{c}3-1 \\ 2\end{array}\right)+1$.

Lemma 9. Let $C$ be any maximal clique of a graph $G \in \mathcal{F}$ orb $\left(K^{-1}\left(\mathcal{F}_{p}\right)\right)$. There exists a family $\mathcal{F}=\left\{C_{1}, C_{2}, \ldots, C_{p}\right\}$ of maximal cliques of $G$ such that $C=C_{1}=I \cup D \cup I^{\prime} \cup D^{\prime}$.

Proof. If $C$ belongs to any family of $p$ pairwise intersecting maximal cliques of $G$, then the proof follows from Lemma 4. Thus, assume there is no such a family. Let $\mathcal{F}=\left\{C_{1}, C_{2}, \ldots, C_{p}\right\}$ be a family of pairwise intersecting maximal cliques of $G$. Clearly, $C \notin \mathcal{F}$, and without loss of generality, we can assume $C \cap C_{1}=\varnothing$ and $C \cap C_{2}=\varnothing$. In addition, by Theorem 5 , each vertex of $C$ is the exact intersection or the exact difference between two members of $\mathcal{F}$. We claim that every vertex of $C$ is the exact intersection or the exact difference between two members of the family $\mathcal{F}=\left\{C, C_{2}, \ldots, C_{p}\right\}$. Indeed, let $C_{i}$ and $C_{j}$ in $\mathcal{F}$ be such that $\{x\}=C_{i} \cap C_{j}$ or $\{x\}=C_{i} \backslash C_{j}$. Notice that $C_{i} \neq C_{1}$. In the first case, clearly $C_{j} \neq C_{1}$; thus $C_{i}$ and $C_{j}$ belong to $\mathcal{F}^{\prime}$. In the second case, if $C_{j}=C_{1}$, then $\{x\}=C_{i} \backslash C_{1}=C \cap C_{i}$ because $C_{1} \cap C=\varnothing$; hence $\{x\}$ is the exact intersection between two members of $\mathcal{F}^{\prime}$.

Theorem 10. Let $G \in \mathcal{F}$ orb $\left(K^{-1}\left(\mathcal{F}_{p}\right)\right)$. If $p \geq 4$ then every maximal clique of $G$ contains at most $\left(\begin{array}{c}p-1 \\ 2\end{array}\right)+1$ vertices.

Proof. Follows from Lemmas 8 and 9. 


\section{3 | CONCLUDING REMARKS}

Ramsey numbers provide another way to conclude that $\mathcal{F}$ orb $\left(K^{-1}\left(\mathcal{F}_{p}\right)\right)$ is finite. Let $G \in \mathcal{F}$ orb $\left(K^{-1}\left(\mathcal{F}_{p}\right)\right)$, for $p \geq 4$. Corollary 6 tells us that the stability number of $G$ is at most $p$, whereas Theorem 10 tells us that the clique number of $G$ is at most $\left(\begin{array}{c}p-1 \\ 2\end{array}\right)+1$. Consider the Ramsey number $r\left(\left(\begin{array}{c}p-1 \\ 2\end{array}\right)+2, p+1\right)=k$. This means that every graph with at least $k$ vertices has a clique of size $\left(\begin{array}{c}p-1 \\ 2\end{array}\right)+2$ or a stable set of size $p+1$. Thus, $G$ must contain at most $k-1$ vertices, that is, $\mathcal{F}$ orb $\left(K^{-1}\left(\mathcal{F}_{p}\right)\right)$ is finite.

Ramsey numbers provide loose upper bounds for the number of vertices of a graph in $\mathcal{F}$ orb $\left(K^{-1}\left(\mathcal{F}_{p}\right)\right)$. For instance, a graph $G \in \mathcal{F}$ orb $\left(K^{-1}\left(\mathcal{F}_{4}\right)\right)$ must contain four pairwise intersecting maximal cliques and its number of vertices is bounded according to the inequalities $|V(G)| \leq r\left(\left(\begin{array}{c}4-1 \\ 2\end{array}\right)+2,4+1\right)-1=r(5,5)-1 \leq 48$. However, by Corollary 6 , we have $|V(G)| \leq 3\left(\begin{array}{l}4 \\ 2\end{array}\right)=18$; and, by Theorem 2, each graph in $\mathcal{F}_{0}$ rb $\left(K^{-1}\left(\mathcal{F}_{4}\right)\right)$ has at most seven vertices. Hence, an interesting question is how to obtain better upper bounds for the number of vertices of a graph in $\mathcal{F}$ orb $\left(K^{-1}\left(\mathcal{F}_{p}\right)\right)$.

Although $\mid \mathcal{F}$ orb $\left(K^{-1}\left(\mathcal{F}_{p}\right)\right) \mid$ seems to be exponential in $p$, another interesting question is to know whether it is possible to devise a systematic method for constructing $\mathcal{F}$ orb $\left(K^{-1}\left(\mathcal{F}_{p+1}\right)\right)$ from $\mathcal{F} \operatorname{orb}\left(K^{-1}\left(\mathcal{F}_{p}\right)\right)$ by the addition of new structures to each graph $G$ in $\mathcal{F} o r b\left(K^{-1}\left(\mathcal{F}_{p}\right)\right)$ in all possible ways, to obtain vertex-minimal graphs $G^{\prime}$ such that $\omega\left(K\left(G^{\prime}\right)\right) \geq p+1$.

\section{ACKNOWLEDGMENT}

Claudia L. Sales is partially supported by CNPq, Brazil. Fabio Protti is partially supported by CNPq and FAPERJ, Brazil.

\section{ORCID}

Fabio Protti (D) http://orcid.org/0000-0001-9924-0079

\section{REFERENCES}

1. L. Alcón, Clique-critical graphs: Maximum size and recognition, Discrete Appl. Math. 154 (2006), 1799-1802.

2. L. Alcón and M. Gutierrez, Clique graphs of planar graphs, Ars Comb. 71 (2004), 257-265.

3. L. Alcón, L. Faria, C. M. H. de Figueiredo, and M. Gutierrez, The complexity of clique graph recognition, Theor. Comp. Sci. 410 (2009), 2072-2083.

4. R. C. Hamelink, A partial characterization of clique graphs, J. Comb. Theory 5 (1968), 192-197.

5. S. T. Hedetniemi and P. J. Slater, Line graphs of triangleless graphs and iterated clique graphs, Graph Theory and Applications, Springer, 1972, pp. 139-147.

6. S. M. Hedge and Suresh Dara, On clique convergence of graphs. AKCE International, J. Graph Comb 13 (2016), no. 3, 261-266.

7. J. M. Lewis and M. Yannakakis, The node-deletion problem for hereditary properties is NP-Complete, J Comp Syst Sci 20 (1980), 219-230.

8. C. L. Lucchesi, C. P. Mello, and J. L. Szwarcfiter, On clique-complete graphs, Discrete Math. 183 (1998), 247-254.

9. V. Neumann-Lara, On clique-divergent graphs, Problems Combinatoires et Théorie des Graphes, Colloques Internationaux du CNRS, Paris, vol. 260, 1978, pp. 313-315.

10. E. Prisner, Graph dynamics. Pitman Research Notes in Mathematics SeriesLongman, Harlow, 1995, p. 338. 
11. F. Protti, F. M. G. França, and J. L. Szwarcfiter, On computing all maximal cliques distributedly, Solving irregularly structured problems in parallel (IRREGULAR 1997). Lecture Notes in Computer Science 1253 (G. Bilardi, A. Ferreira, R. Lüling, and J. Rolim, eds.), Springer, Berlin, Heidelberg, 1997, pp. 37-48.

12. F. Protti and J. L. Szwarcfiter, Clique-inverse graphs of $\mathrm{K}_{3}$-free and $\mathrm{K}_{4}$-free graphs, J. Graph Theory 35 (2000), 257-272.

13. F. Protti and J. L. Szwarcfiter, On clique graphs with linear size, Congr. Numer. 143 (2000), 207-219.

14. F. Protti and J. L. Szwarcfiter, Clique-inverse graphs of bipartite graphs, J. Comb. Math. Comb. Comput. 40 (2002), 193-203.

15. F. S. Roberts and J. H. Spencer, A characterization of clique graphs, J. Comb. Theory Series B 10 (1971), 102-108.

16. J. L. Szwarcfiter and C. F. Bornstein, Clique graphs of chordal and path graphs, SIAM J. Discrete Math. 7 (1994), 331-336.

17. J. Szwarcfiter, A survey on clique graphs, Recent advances in Algorithms and Combinatorics (B. Reed, and C. LinharesSales, eds.), Springer, 2003, pp. 109-136.

How to cite this article: Alcón L, Gravier S, Sales CL, Protti F, Ravenna G. On cliqueinverse graphs of graphs with bounded clique number. J Graph Theory. 2020;94:531-538. https://doi.org/10.1002/jgt.22544 